\title{
Protective Measures against X-rays and Radium.
}

A COMMITTEE was recently formed in A London to see whether some general precautionary measures could be outlined which would be of service to those employed in the use of X-rays or radium for medical, scientific, or industrial purposes. The members of the committee are as follows: Sir Humphry Rolleston (chairman), Sir Archibald Reid, Dr. Robert Knox, Dr. G. Harrison Orton, Dr. S. Gilbert Scott, Dr. J. C. Mottram, Dr. G. W. C. Kaye, and Mr. Cuthbert Andrews. Dr. Stanley Melville and Prof. S. Russ are acting as honorary secretaries to the committee. The need for a statement on this subject has been felt for some time. During the war the Röntgen Society issued a printed card pointing out the dangers of exposing parts of the body to X-rays unduly, but the uses of these forms of radiation are becoming so numerous in medicine and the arts that it was felt that the ground should be gone over in more detail, and general recommendations drawn up as to the conditions under which work of this character should be carried out.

The preliminary report of the committee has just been issued. It is a carefully thought-out statement of present knowledge in regard to the equipment, ventilation, and working conditions of $\mathrm{X}$-ray and radium departments. We are glad to see from the introduction to the report that the committee holds the view that the dangers which may attend the use of these radiations can be avoided entirely by the provision of efficient protection and suitable working conditions.

The damage which people have suffered in the past falls into two categories:-

(I) Visible injuries to the superficial tissues which may result in permanent damage.

(2) Derangements of internal organs and changes in the blood. These are especially important, as their early manifestation is often unrecognised.

The protective measures to be employed naturally vary with the work in hand, and the report contains details of the measures which the committee thinks appropriate to (I) X-rays for diagnostic purposes, (2) X-rays for superficial therapy,
(3) X-rays for deep therapy, (4) X-rays for industrial and research purposes, (5) electrical precautions in X-ray departments, (6) ventilation of $\mathrm{X}$-ray departments, and ( 7 ) radium therapy.

The report concludes with a statement bearing upon several aspects of the subject, and we accordingly reproduce it in full :

"The governing bodies of many institutions where radiological work is carried on may wish to have further guarantees of the general safety of the conditions under which their personnel work.

"(I) Although the committee believe that an adequate degree of safety would result if the recommendations now put forward were acted upon, they would point out that this is entirely dependent upon the loyal co-operation of the personnel in following the precautionary measures outlined for their benefit.

"(2) The committee would also point out that the National Physical Laboratory, Teddington, is prepared to carry out exact measurements upon $\mathrm{X}$-ray protective materials, and to arrange for periodic inspection of existing installations on the lines of the present recommendations.

"(3) Further, in view of the varying susceptibilities of workers to radiation, the committee recommend that wherever possible periodic tests - e.g. every three months-be made upon the blood of the personnel, so that any changes which occur may be recognised at an early stage. In the present state of our knowledge it is difficult to decide when small variations from the normal blood-count become significant."

It is satisfactory to learn that the committee intends to continue to meet and to consider the advisability of directing some researches which arise out of the considerations involved in the memorandum in question.

Suggestions and offers of personal or other assistance are invited; they should be forwarded to the honorary secretaries of the X-ray and Radium Protection Committee, from whom copies of the preliminary report may be obtained, c/o Royal Society of Medicine, Wimpole Street, W.I.

\section{Cosmogony and Stellar Evolution. ${ }^{1}$}

\section{By J. H. Jeans, Sec.R.S.}

\section{I.-The Evolution of Gaseous Masses.}

THE progress of observational astronomy has 1 made it abundantly clear that astronomical formations fall into well-defined classes; they are almost "manufactured articles" in the sense in which Clerk Maxwell applied the phrase to atoms. Just as atoms of hydrogen or calcium are believed to be of similar structure no matter where they are found, so star-clusters, spiral nebulæ, binary stars are seen to be similar, although in

1 Lectures delivered at King's College on May 3 and ro. No. 2696 , VOL. IO7] a less degree, no matter in what part of the sky they appear. The problem of cosmogony is to investigate the origins of these comparatively uniform formations and the process of transition from one class to another.

In attacking this problem the cosmogonist of to-day stands upon the shoulders not only of previous cosmogonists, but also, what is of even greater importance, upon the shoulders of the brilliant and industrious astronomical observers of the past century. We shall find it convenient 
to take as our starting point the most famous theory of cosmogony ever propounded-the nebular hypothesis of Laplace-and we shall examine to what extent it remains tenable in the light of modern observational and theoretical research.

Laplace's hypothesis referred primarily to the genesis of the solar system, which he believed to have originated out of a hot nebulous mass that shrank as it cooled. The nebula was supposed to be in rotation, so that the principle of conservation of angular momentum required that as the mass cooled its speed of rotation should increase. It is well known that a mass either of gas or of liquid in rotation cannot rest in equilibrium in the spherical shape which would be assumed in the absence of rotation. If the rotation is very slow the equilibrium shape will be an oblate spheroid of small eccentricity. As the rotation increases, the ellipticity will increase, but it is found that the spheroidal shape is soon departed from. Laplace believed, as a matter of conjecture rather than of reasoned proof, that with continually increasing rotation a mass of gas would in time reach a stage at which it could no longer exist as a single continuous mass. When this stage was reached he believed that a ring of particles would be discharged from the equator through the centrifugal force of rotation outweighing the centripetal force of gravitation. The mathematical researches of Roche ( 1873 ) provided some support for this general conjecture, and more recent investigations put its general accuracy beyond doubt.

It is found that the changes of shape which accompany increase of rotation are, in their general features, the same for all masses, whether gaseous or fluid, provided only that there is sufficient central condensation of mass. When the rotation becomes so great that the spheroidal figure is departed from, the equator of the mass is found to pull out into a pronounced edge which ultimately becomes perfectly sharp (see Fig. I). The mass has now assumed a lenticular shape, and any further increase of rotation results in matter being discharged from this sharp edge. The lenticular shape is retained from now on, the sharp edge acting like a safety valve and emitting just so much matter as is necessary to carry off the excess of angular momentum beyond the maximum which can be carried by the central mass. Fig. I shows the configurations of the lenticular figures for masses of gas in adiabatic equilibrium, in which $\gamma$ (ratio of specific heats) has the extreme values $x \cdot 2$ and $2 \cdot 2$ respectively. Other calculated lenticular figures show generally similar shapes. With a further increase of rotation beyond that for which these curves are drawn, the figures would remain unaltered save for the addition of a distribution of matter in the equatorial plane-the matter already thrown off from the sharp edge of the lens.

If gaseous stars assume these forms our telescopes refuse to reveal them. Even in the most powerful telescopes the stars remain infinitesimal points of light; the only bodies which show any observable shape are the nebulæ. It is highly significant that a number of these exhibit precisely the lenticular shape just described. This is in most cases accompanied by a distribution of matter in the plane through the sharp edge of the lens. A number of such nebulæ have been found by direct spectroscopic observation to be in rotation about an axis perpendicular to this plane. Thus there is very strong justification for supposing that these nebulæ are masses of gas or other matter with high central condensation behaving precisely as imagined by Laplace-rotating and throwing off their excess of angular momentum as they cool by the ejection of matter in their equatorial planes.

There is, however, almost incontrovertible evidence that the nebulæ which have just been described are nothing but ordinary spiral nebulæ seen edgewise, for observation discloses a continuous sequence of nebulæ the shapes of which bridge completely the gap between the lenticular nebulæ, in which we are looking at right angles to the axis of rotation, and the familiar spiral nebula in which we look approximately along this axis. The characteristic nebula shows a nucleus which we can now identify with the lenticular
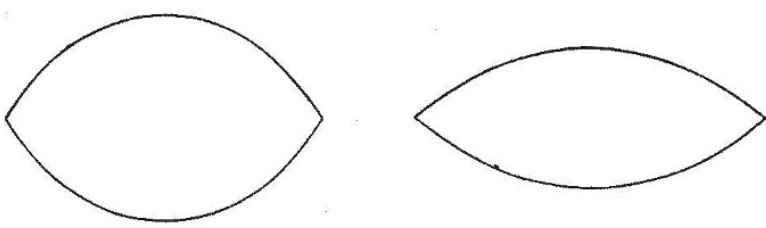

Frg. x.-Figures of equilibrium for rotating masses of gas.

figure demanded by theory, having two arms emerging symmetrically from opposite points of the nucleus. If our identification is correct these arms must be formed out of the matter already discharged from the nucleus. It has in point of fact been found by van Maanen and Kostinsky that the matter in the arms appears to be in motion approximately along the arms and in the outward direction.

Any external gravitational field, whether of the universe as a whole or of neighbouring stars or nebulæ, would produce a tidal field similar to that produced by the sun and moon on the surface of our earth, a field specified mathematically by a second harmonic. This field, no matter how small in amount, would suffice to destroy the exact circular shape of the "equator" of the nucleus and so would concentrate the emission of matter at two opposite points on this equator. Thus it is easy to understand why the nebulæ, as a rule, exhibit two symmetrical arms emerging from antipodal points. It is very much less easy to understand why these arms should be of the universal spiral form-the absence of any explanation of this form must be regarded as a serious drawback to our interpretation of the spiral nebulæ. It is readily proved that the ejected filaments of matter, whatever the shape they assume, could not remain of uniform line-density. 
Such a distribution of density would be unstable, and it can be proved that nuclei would form at .approximately equal distances, around which the matter of the arms would condense. In this way it is possible to explain the nuclei and condensations which are observed in the arms of the spiral nebulæ. It is also found possible to calculate the amount of matter which will condense around each nucleus; the mass of each is found to be of the order of magnitude of the known masses of the stars.

In this way I have been led to conjecture that the spiral nebulæ are whirling masses of gas which, owing to their rapidity of rotation, throw off gaseous stars much as a "Catherine-wheel" firework throws off sparks. If so, the condensations in the arms of these nebulæ are stars in the process of birth. Dynamically the mechanism is almost identical with that imagined by Laplace as resulting in the birth of systems of planets and satellites, but on a far more stupendous scale. The final product of the chain of events we have been considering must be some type of starcluster-perhaps a globular star-cluster, or possibly an "island-universe" similar to our galactic system. The difficulties in the way of an exact mathematical investigation into the history of the ejected gas as the filaments condense around nuclei and as these form stars and begin to move as detached bodies are enormous. On the other hand, the determination of the final steady states possible for a system of stars created in this way is quite simple. There is found to be only one type of final steady state possible for a system of stars created out of a rotating mass of gas, and this shows exactly the features presented by the system of stars of which our sun is a member. The system of stars will be of a flattened shape, symmetrical about the plane of greatest crosssection (the galactic plane in our system); the velocities in any small region of space will not be distributed at random, but will show a preference for two opposite directions ("star-streaming "); these directions will be parallel to the plane of symmetry and perpendicular to the radius to the centre of the system. This last direction is that given by Charlier for the direction of "star-streaming" in our system. Our system passes all tests for having been born out of a spiral nebula the plane of which was what is now the plane of the Milky Way; indeed, Easton and others have claimed to find traces of the two spiral arms still surviving in the distribution of stars in this plane, as though the final steady state had not yet been reached.

Let us now turn to a study of the lives of individual stars. To the naked eye the stars appear as mere points of light of varying brightness. The telescope adds little except possibly differences of colour. The spectroscope appears at first to add a wealth of new information, but a detailed study of stellar spectra discloses the unexpected fact that all stellar spectra, apart from a few exceptions, fall into one single linear series. Photographs of the spectra of all stars, in which varying exposures have been made to compensate for varying brightnesses, can be arranged uniquely in a consecutive order in which each spectrum differs only imperceptibly from its neighbour. All the complicated diversities of stellar spectra appear to be determined, in the main, by one single variable. This is believed, with good reason, to be the temperature of the star's surface.

Positions on this linear series are specified by reference to six selected points denoted by the letters $B, A, F, G, K, M$ in this order. The order given is that of decreasing surface temperature. Stars having B-spectra are of bluish colour with a surface temperature of $10,000^{\circ} \mathrm{C}$. or more. Stars of type $M$ are red with a surface temperature of only $3000^{\circ} \mathrm{C}$. Our sun is of type G, with a surface temperature of about $6000^{\circ} \mathrm{C}$.

We might also arrange the stars in order of brightness. The distances of many stars are known, and for these we can calculate the "absolute brightness" or "luminosity"-i.e. the amount of light emitted as compared with our sun. Since the masses of the stars are all approximately the same, it might be expected that the order of "luminosity" would prove to be substantially the same as that of surface temperature, but this does not prove to be the case. Eight years ago it was found by Hertzsprung and $H$. N. Russell that the red M-stars fell into two widely different classes, one class having abnormally high luminosity, and the other abnormally low. The ratio of luminosities in the two classes is of the order of 10,000 to $I$, and since the surface temperatures are the same, this ratio must imply a corresponding ratio in the areas of the radiating surfaces. Thus the two classes of M-stars must have volumes in a ratio of about $x, 000,000$ to one, for which sufficient reason they have been designated "giants" and "dwarfs." From a comprehensive discussion by Russell, recently confirmed by Adams and Joy, it is clear that the demarcation between "giants" and "dwarfs" extends, although with diminished intensity, through the types $\mathrm{K}, \mathrm{G}$, and $\mathrm{F}$, while at types $\mathrm{A}$ and $\mathrm{B}$ the classes coalesce.

Lately Shapley, by determining the distances of the globular clusters, has greatly increased our knowledge of stellar luminosities, and has calculated the individual luminosities of II 52 giant stars in clusters. If we plot the logarithms of the luminosity (or the absolute magnitude) against spectral type as in Fig. 2, the vast majority of Shapley's I $_{52} 2$ stars are found to lie within the belt marked "giants," while of the stars previously discussed by Russell and by Adams and Joy nearly all lie either within this belt or within that marked "dwarfs." In this diagram a few typical stars have been marked. The stars $\alpha$ Orionis and our near neighbour Lalande $2 \mathrm{I} .185$ are examples of giant and dwarf red stars. The diameter of the former has recently been found by direct measurement to be about 300 times that of our sun, corresponding to a density of the order of at most one-thousandth of that of atmospheric air; the latter has a luminosity only 0.009 times that of the sun, and probably a mean density comNo. 2606 , vor.. то7 7 
parable with that of the earth. Our sun and our nearest stellar neighbour, $\alpha$ Centauri, are marked as typical dwarfs of type G, and Sirius is a representative A-type star.

From the known luminosity and surface temperature of any star it is easy to calculate its surface and so its density. Giants of types $G$ and $\mathrm{K}$ are found to have densities of the order of 0.004 and 0.0005 respectively, agreeing with the known densities of binary stars of these types. Sirius, with a luminosity of forty-eight times, and a surface temperature about one and a half times, those of our sun, must have a surface nine times as great. Its mass is 3.4 times the solar mass, so that its density must be about $\mathbf{0 \cdot 2}$. In general it is found that all giant stars must be gaseous, of

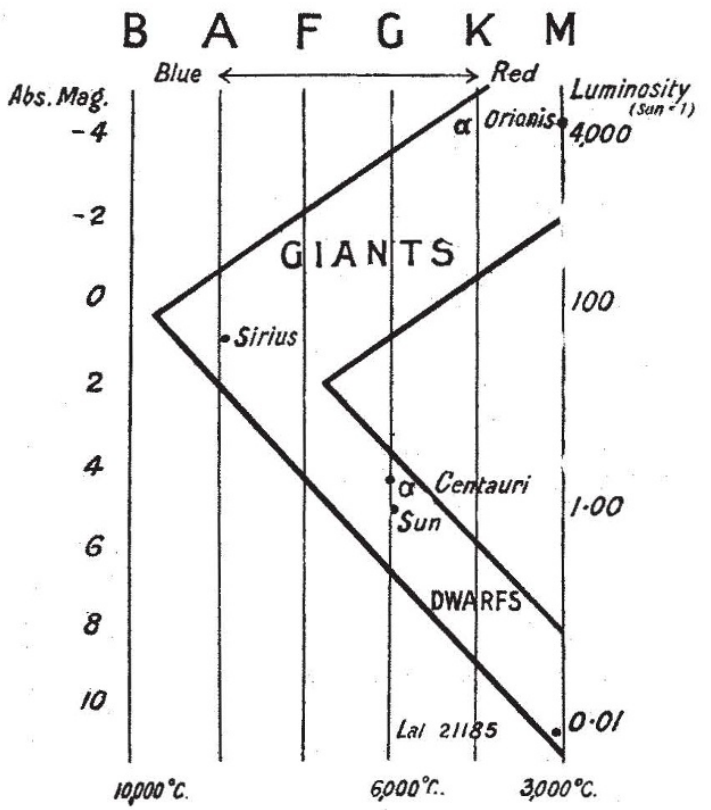

FIG. 2.-Luminosity-temperature diagram.

density so low that the ordinary gas-laws will be approximately obeyed. Dwarf stars may be gaseous or liquid or solid, but, if gaseous, they are so dense that the gas-laws will be nowhere near the truth. It is now easy to see why, in the giant stars, increase of temperature and density go together; this is merely a consequence of Lane's law. But the dwarfs may be thought of as approximating rather to masses of fixed dimensions, and for these the luminosity falls off as the temperature decreases.

Our sun radiates light at a rate of about 2 ergs per second per gram of its mass. Gravitational contraction, as Lord Kelvin showed, could provide energy at this rate for only about $20,000,000$ years, and radio-active and chemical energy could only slightly lengthen this period. For a giant star, radiating at 1000 times the rate of the sun, the maximum period would be only a few thousand years. This period is far too short, and it is now generally accepted that, so far from gravitation and known sources of energy providing the whole of a star's radiation, they can provide only an insignificant fraction. Energy of adequate amount can originate only from sub-atomic sources, as, for instance, from internal rearrangements in the positive nuclei of the atoms or from the transformation of a small fraction of the star's mass into energy. It is a matter of simple calculation to show that all other stores of energy in a star can constitute only an insignificant reservoir of energy which, unless continually replenished from sub-atomic sources, would be exhausted in, astronomically, a moment. Thus the rates of radiation and of generation of sub-atomic energy must be practically equal, and the luminosity of a star will be determined by the latter rate at any instant.

We may now think of the evolution of the stars as represented by the march of a vast army through our diagram (Fig. 2), the individuals keeping, for the most part, within the marked belt. Each individual takes his marching orders from the supply of sub-atomic energy, and so long as we remain in ignorance of the exact source and nature of this we cannot be certain whether the motion of the army is up or down, or even that it is all in the same direction. But if we are right in conjecturing that the stars were born out of a nebula of very low density, the order of march will be from low density to high; our army will be marching downwards in the diagram. Its tail, except for a few stragglers, is about at absolute magnitude -4 , its head is lost in darkness. In the next lecture we must study the incidents which may occur during the march of this army of stars.

\section{(To be continued.)}

\section{Obituary.}

Dr. A. M. Kellas.

$\mathrm{B}^{\mathrm{Y}}$ the death of Dr. A. M. Kellas we have lost one of the best authorities on the effect of high altitudes on the human system. No one else had so great a practical knowledge, or worked scientifically at the subject with more persistence than he.

Born in Aberdeen, he was educated there, and afterwards went to Edinburgh, London, and Heidelberg. For some time he was assistant to
Sir William Ramsay, and afterwards lecturer on chemistry at Middlesex Hospital.

As a teacher he was most successful, taking endless trouble in helping backward students. In pure chemistry he did little research, his chief contribution being a long and careful investigation on "The Determination of the Molecular Complexity of Liquid Sulphur," published in I9r8. But during the last ten years he gave up most of his spare time to study the physiological and physical NO. 2696 , VOL. IO7] 